\title{
Non-LTE Models for Neutron Star Atmospheres and Supernova-Fallback Disks
}

\author{
K. Werner ${ }^{a}$, T. Nagel ${ }^{\text {a }}$, T. Rauch ${ }^{\mathrm{a}}$, and V. Suleimanov ${ }^{\mathrm{a}, \mathrm{b}}$ \\ ${ }^{a}$ Institut für Astronomie und Astrophysik, Universität Tübingen, Sand 1, 72076 \\ Tübingen, Germany

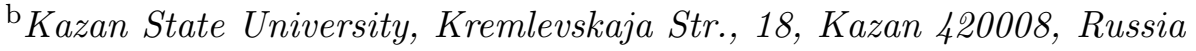

\begin{abstract}
We describe our recent progress in modeling supernova-fallback disks and neutron star (NS) atmospheres.

We present a first detailed spectrum synthesis calculation of a SN-fallback disk composed of iron. We assume a thin disk with a radial structure described by the $\alpha$-disk model. The vertical structure and emission spectrum are computed selfconsistently by solving the structure equations simultaneously with the radiation transfer equations under non-LTE conditions. We describe the properties of a specific disk model and discuss various effects on the emergent UV/optical spectrum.

We investigate Compton scattering effects on the thermal spectrum of NSs. In addition, we constructed a new generation of metal line-blanketed non-LTE model atmospheres for NSs. It is compared to X-ray burst spectra of EXO 0748-676. It is possible that the gravitational redshift, deduced from absorption lines, is lower $(z=0.24)$ than hitherto assumed $(z=0.35)$. Accordingly, this would result in NS mass and radius lower limits of $M \geq 1.63 \mathrm{M}_{\odot}$ and $R \geq 13.8 \mathrm{~km}$.
\end{abstract}

Key words: Neutron stars, Infall, accretion, and accretion disks PACS: 95.30.Jx, 97.60.Jd, 98.35.Mp

\section{Introduction}

We report on our recent progress in radiation transfer modeling for supernovafallback disks and non-magnetic neutron star (NS) atmospheres. Disk modeling is described in Sect. 2. We then present effects of Compton scattering on

Email address: werner@astro.uni-tuebingen.de (K. Werner). 
the thermal spectra of NS atmospheres (Sect. 3). In Sect. 4 we describe the construction of new line-blanketed NLTE atmosphere models for NSs. These models are applied to X-ray burst spectra of EXO 0748-676 (Sect. 5).

\section{NLTE models for SN fallback disks}

Anomalous X-ray pulsars (AXPs) are slowly rotating young isolated NSs. Their X-ray luminosities greatly exceed the rates of rotational energy loss. It is now generally believed that AXPs are magnetars and that their X-ray luminosity is powered by magnetic energy. As an alternative explanation the $\mathrm{X}$-ray emission was attributed to accretion from a disk that is made up of supernova-fallback material (e.g. van Paradijs et al., 1995). It is still a matter of debate whether the disk model is appropriate. For example, the discovery of optical pulsations in $4 \mathrm{U} 0142+61$ with the same period like the X-ray pulsations (Kern \& Martin, 2002) appears to be a strong argument against the disk model. It was argued that reprocessing of the pulsed NS X-ray emission in the disk cannot explain the high optical pulsed fraction, because disk radiation would be dominated by viscous dissipation and not by reprocessed NS irradiation (Kern \& Martin, 2002). In contrast, Ertan \& Cheng (2004) showed that the optical pulsations can be explained either by the magnetar outer gap model or by the disk-star dynamo model. A spectral break in the optical spectrum of 4U 0142+61 was discovered by Hulleman et al. (2004) and also taken as an argument against the disk model. The recent discovery of mid-IR emission from this AXP (Wang et al., 2006), however, has strongly rekindled the interest in studies of fallback-disk emission properties. The emission was attributed to a cool, passive dust debris disk, but it was shown by Ertan et al. (2006) that it can be explained by an active, dissipating gas disk.

The emission from fallback disks was hitherto modeled with blackbody spectra. In view of the importance of disk models for the quantitative interpretation of observational data it is highly desirable to construct more realistic models by detailed radiation-transfer calculations. We employ our computer code AcDc (Nagel et al., 2004). For a detailed description of the calculation of fallback disk models we refer to Werner et al. (2006). The radial disk structure is calculated assuming a stationary, Keplerian, geometrically thin $\alpha$-disk. For the results reported here we selected the following model parameters. The NS mass is $1.4 \mathrm{M}_{\odot}$. The radii of the inner and outer disk edges are 2000 and $200000 \mathrm{~km}$, respectively. The accretion rate was set to $\dot{M}=3 \cdot 10^{-9} \mathrm{M}_{\odot} / \mathrm{yr}$, which is a relatively large value. It is motivated by the fact, that this corresponds to the upper limit for the non-detection of UV radiation from a possible disk around SN 1987A under the assumption of an outer disk radius of $100000 \mathrm{~km}$ (Graves et al., 2005). Our code allows for the irradiation of the

disk by the central source, however, the results presented here are computed 


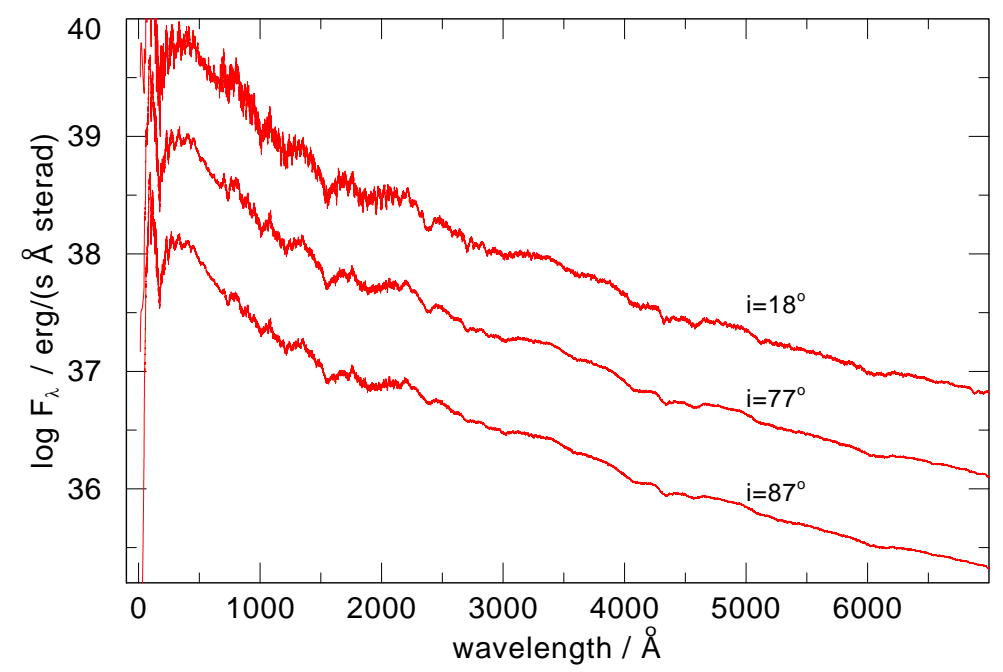

Fig. 1. Spectrum of a fallback-disk model seen under three inclination angles $i$. Broad iron-line blends are detectable even in the almost edge-on case.

with zero incident intensity. The disk is represented by nine rings. The vertical structure of each ring is determined from the simultaneous solution of the radiation transfer equations plus the structure equations (radiative and hydrostatic equilibrium). They also consist of the non-LTE rate equations for the atomic population densities. The chemical composition of SN fallback material is not exactly known. It depends on the amount of mass that goes into the disk. A disk with a small mass ( say $\leq 0.001 \mathrm{M}_{\odot}$ ) will be composed of siliconburning ash (Menou et al., 2001). For simplicity we assume a pure-iron disk composition but also tested a composition that represents silicon-burning ash. It contains iron ( $80 \%$ mass fraction) as well as silicon and sulfur (10\% each). Fig. 1 displays the overall disk spectrum. We summarize the model properties as follows:

- Depending on the inclination, the disk flux can be a factor of two higher or lower compared to a blackbody radiating disk at the same inclination.

- Strong iron line blanketing causes broad (>100 ̊) spectral features that could be detectable even from almost edge-on disks.

- Limb darkening affects the overall disk spectrum (in addition to the geometric foreshortening factor). Depending on inclination and spectral band, the disk intensity varies up to a factor of three.

- The overall disk spectrum is independent of the detailed chemical composition as long as $\mathrm{Fe}$ is the dominant species. In particular, a pure-Fe composition is spectroscopically indistinguishable from Si-burning ash.

- The overall disk spectrum is hardly influenced by non-LTE effects, however, equivalent widths of individual line blends can change by a factor of two. 


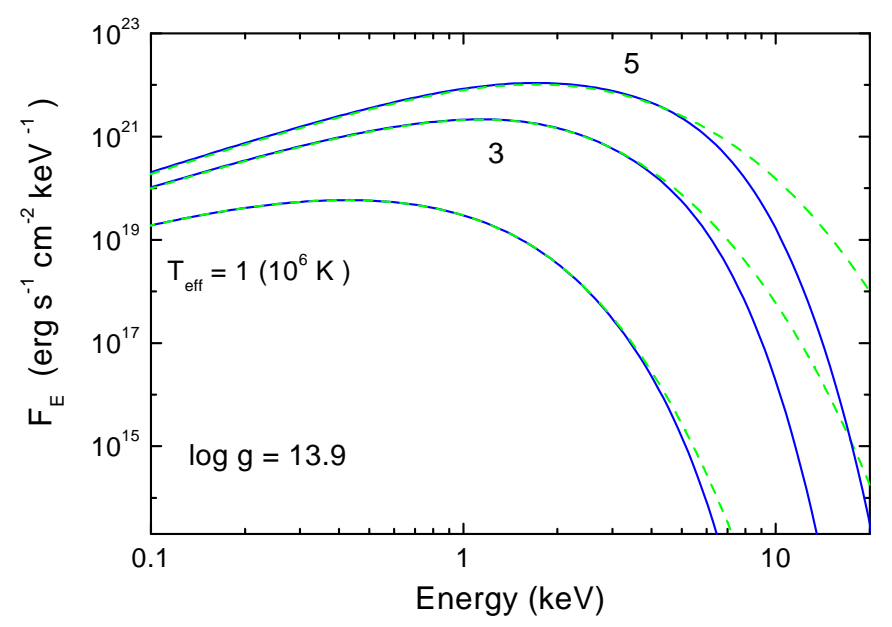

Fig. 2. Emergent spectra of three pure-H models with different $T_{\text {eff }}\left(1,3\right.$, and $\left.5 \cdot 10^{6} \mathrm{~K}\right)$ computed with Thomson (dashed lines) and Compton (full lines) electron scattering.

\section{Compton scattering in NS model atmospheres}

The nature of isolated NS (INS) surface layers is not exactly known. At some conditions (depending on surface temperature, magnetic field strength and chemical composition) a surface can be solid, liquid, or have a plasma envelope (Lai \& Salpeter, 1997; Lai, 2001). In the last case the envelope can be considered as a NS atmosphere, and the structure and emergent spectrum of this atmosphere can be computed by using stellar model atmosphere methods. Such modeling was performed by many groups and the model spectra were used to fit observed INS X-ray spectra. One of the important results of these works is that spectra of light elements (hydrogen and helium) atmospheres with low magnetic field are significantly harder than the corresponding blackbody spectra. These elements are fully ionised in atmospheres with $T_{\text {eff }} \geq 10^{6} \mathrm{~K}$. Therefore, the true opacity (mainly due to free-free transitions) decreases with photon energy as $E^{-3}$. At high energies electron scattering is larger than the true opacity and photons emitted deep in the atmosphere (where $T>T_{\text {eff }}$ ) escape after few scatterings on electrons. In all previous work concerning INS, coherent (Thomson) electron scattering is considered. As a result, emergent spectra are very hard. But such a situation is very favorable to change the photon energy due to Compton down-scattering.

It is well known that Compton down-scattering determines the shape of emergent model spectra of hotter NS atmospheres with $T_{\text {eff }} \sim 2 \cdot 10^{7} \mathrm{~K}$ close to the Eddington limit (e.g. Pavlov et al., 1991). These model spectra describe the observed X-ray spectra of X-ray bursting NS in low-mass X-ray binaries (LMXBs), and they are close to diluted blackbody spectra with a hardness factor $f_{c} \sim 1.5-1.9$. But models with Compton scattering taken into account were not calculated for relatively cool atmospheres with $T_{\text {eff }}<10^{7} \mathrm{~K}$. There- 
fore, at present time, the effect of Compton scattering on emergent spectra of INS model atmospheres with $T_{\text {eff }}<5 \cdot 10^{6} \mathrm{~K}$ is not well known.

We investigated the effect of Compton scattering on hot $\left(T_{\text {eff }}>10^{6} \mathrm{~K}\right)$ INS atmospheres with weak magnetic fields $\left(B<10^{8} \mathrm{G}\right)$. In order to compute LTE model atmospheres in hydrostatic and radiative equilibrium we solve the radiation transfer equation with the Kompaneets operator. We calculated a set of models with $T_{\text {eff }}$ in the range $1-5 \cdot 10^{6} \mathrm{~K}$, with two values of surface gravity $(\log g=13.9$ and 14.3$)$, and different chemical compositions (Suleimanov \& Werner, 2007) and the results can be summarized as follows:

- Radiation spectra computed with Compton scattering are softer than those computed without Compton scattering at high energies $(E>5 \mathrm{keV})$ for light element (H or He) model atmospheres (Fig. 2).

- The Compton effect is most significant in $\mathrm{H}$ model atmospheres and models with low surface gravity.

- Compton scattering is less important in models with solar abundance of heavy elements.

- The emergent spectra of the hottest $\left(T_{\text {eff }}>3 \cdot 10^{6} \mathrm{~K}\right)$ models can be described by diluted blackbody spectra with hardness factors $\sim 1.6-1.9$.

\section{Metal line blanketed NLTE models for NS atmospheres}

We are computing a new grid of model atmospheres for weakly magnetized

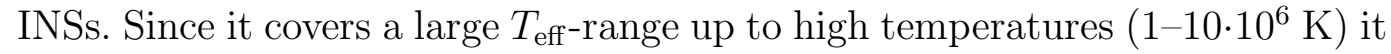
takes into account deviations from LTE for the first time. Werner \& Deetjen (2000) have shown that NLTE effects can affect the temperature structure of the outer photospheric layers. The models are line-blanketed and calculated for different chemical compositions comprising H, He, C, N, O, and iron (Rauch, Suleimanov, Werner, in prep.). We employ the TMAP (Tübingen Model Atmosphere Package) codes (Werner et al., 2003) and account for opacities of millions of iron lines in a statistical way (Rauch \& Deetjen, 2003). The model atoms are constructed using energy levels from NIST and oscillator strengths and photoionisation cross-sections calculated by the Opacity Project (TIPTOPbase). As an example, in Fig. 3 we display the X-ray flux spectra of models with different $T_{\text {eff }}$, solar element composition, and $\log g=14.39$.

\section{Model comparison to X-ray burst spectra of EXO 0748-676}

Cottam et al. (2002) have announced the detection of absorption features in the burst spectra of the LMXB EXO 0748-676. The three most significant 


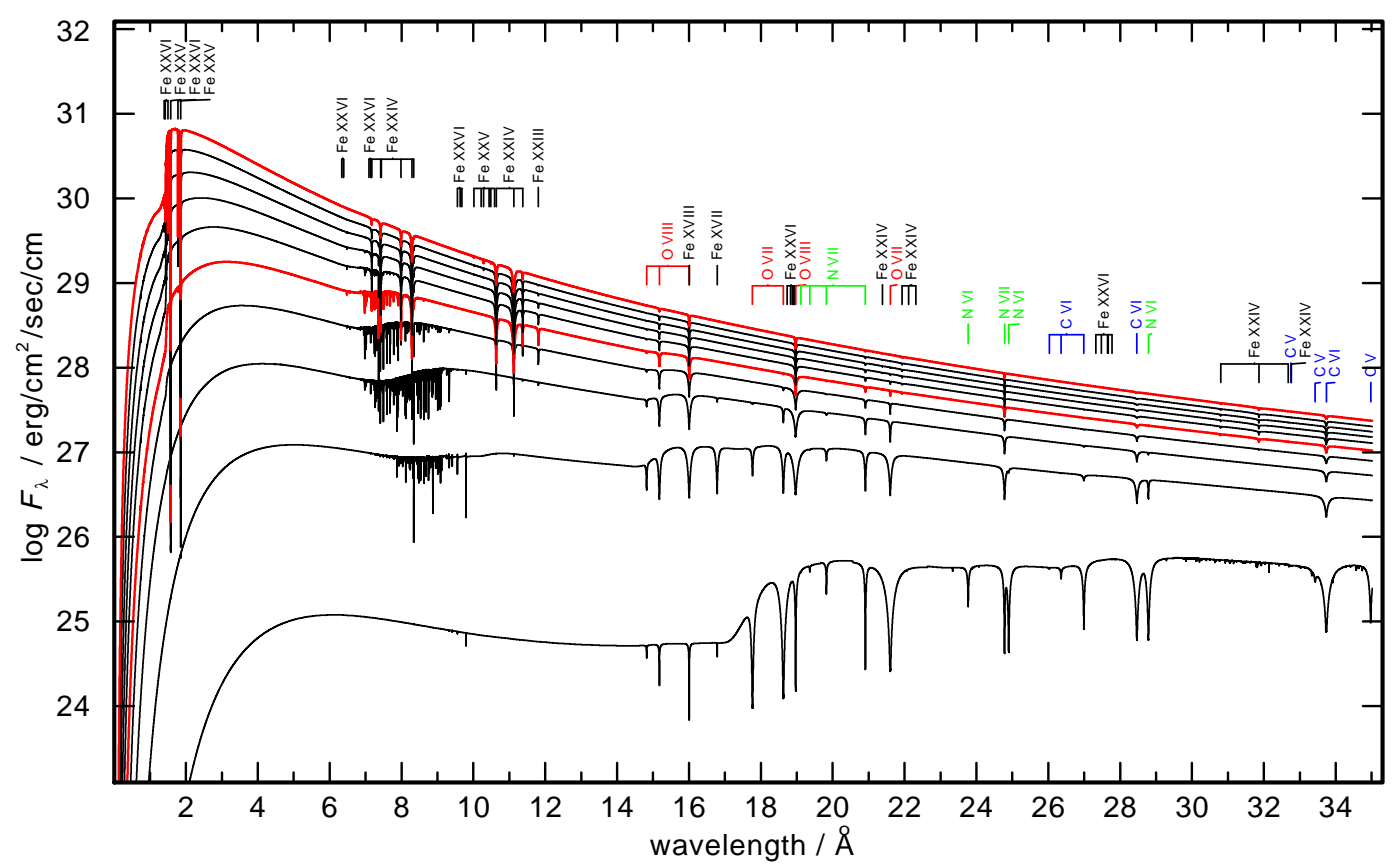

Fig. 3. Spectra of solar composition NLTE model atmospheres with increasing $T_{\text {eff }}$ $\left(1-10 \cdot 10^{6} \mathrm{~K}\right.$ in steps of $\left.10^{6} \mathrm{~K}\right)$ at $\log g=14.39$.

features were identified as photospheric absorption lines, redshifted by $z=0.35$ : A feature at $13.00 \AA$ in the early-burst phases was assigned to the FexxvI $\mathrm{n}=2-3$ transition at $\lambda_{0}=9.5-9.7 \AA$. In the late-burst phase a feature appears at $13.75 \AA$ and it was assigned to the Fexxv $n=2-3$ transition at $\lambda_{0}=10.2 \AA$. A double feature at 25.2/26.0 $\AA$, also appearing in the late-burst phase, was

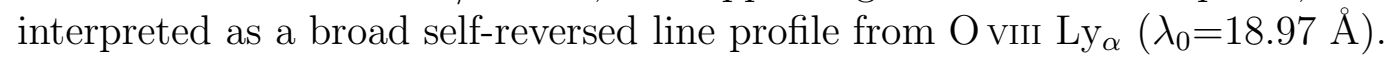

We can use our models to check these line identifications. The upper curve in Fig. 4 is the spectrum of a Fe-dominated (99\%) model with $T_{\text {eff }}=8 \cdot 10^{7} \mathrm{~K}$, $\log g=14.39$, redshifted by $z=0.35$. This Figure can be directly compared to Fig. 1 in Cottam et al. (2002). It is obvious that the aforementioned Fexxvi/ Fe Xxvir lines at (redshifted) $\lambda=13.0 / 13.75 \AA$ are much too weak in the model. In models with different $T_{\text {eff }}$ these lines are even weaker, so that the identification by Cottam et al. (2002) is not confirmed.

Other Fe lines in the models are much stronger and we suggest another possible line identification in the observed spectra. The lower curve in Fig. 4 shows a solar-composition model with the same $T_{\text {eff }}$ and $\log g$ but with a different redshift, namely $z=0.24$. The observed lines at $\lambda=13.0 / 13.75 \AA$ could stem from Fexxiv $\left(\mathrm{n}=2-3, \lambda_{0}=10.6-11.4 \AA\right)$. The $\mathrm{O}$ vin $\mathrm{Ly}_{\alpha}$ line is located at $23.6 \AA$ in the $z=0.24$ redshifted model and a weak feature is seen in the observed spectrum at this location. We would like to stress that our analysis is in a very preliminary stage. But our models clearly indicate that a re-analysis of the X-ray burst spectra of EXO 0748-676 is necessary. 


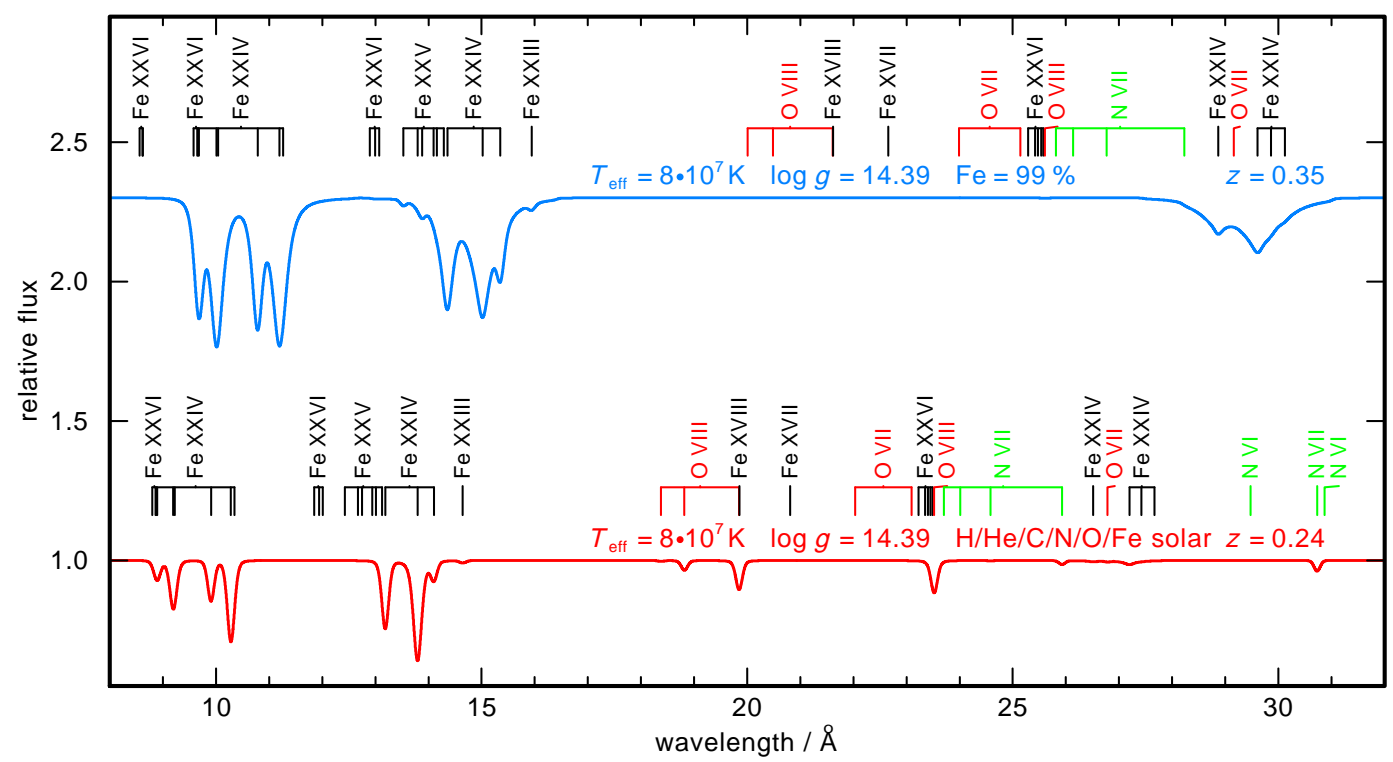

Fig. 4. Spectra from two models with $z=0.35$ (top) and $z=0.24$ (bottom). They suggest that the observed absorption lines in EXO 0748-676 at $\lambda=13.0 / 13.75 \AA$ could stem from Fe XXIV at $z=0.24$ rather than from Fe XXVI/ Fe XXVII at $z=0.35$.

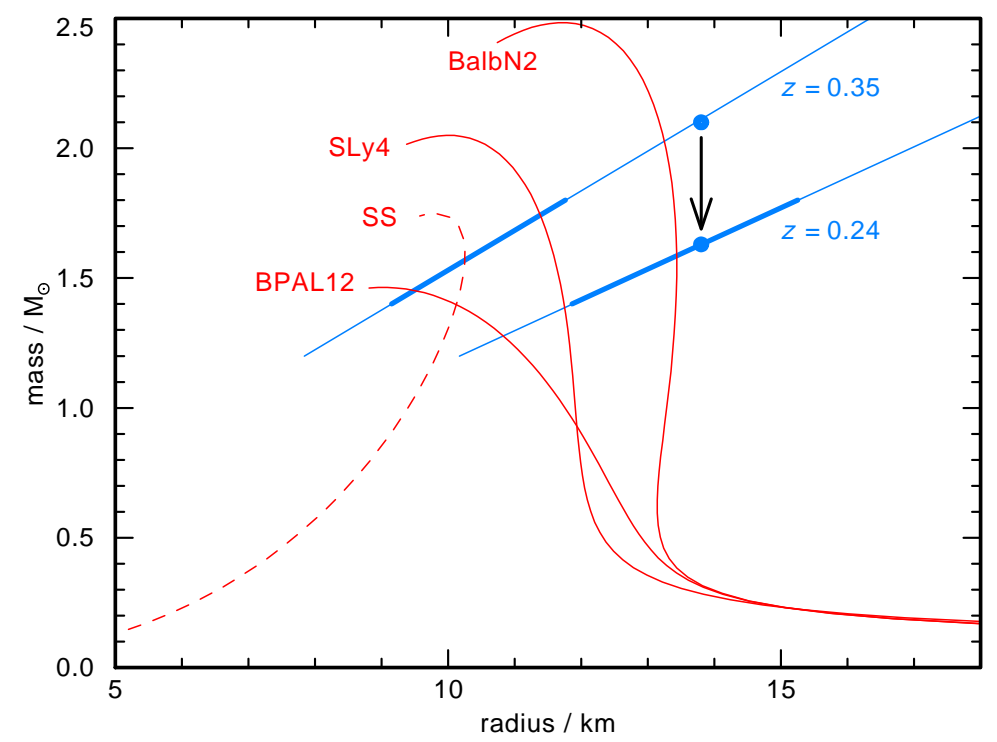

Fig. 5. Allowed values for $M$ and $R$ of EXO 0748-676 for redshifts $z=0.24$ and $z=0.35$ (straight lines; thick portions of the graphs denote the mass range 1.4-1.8 $\mathrm{M}_{\odot}$ ) compared to various theoretical $M-R$ relations (Haensel et al., 2006). The thick dot on the $z=0.35$ line denotes the minimum $M$ and $R$ derived by Özel (2006). The arrow indicates the shift of this result when we assume $z=0.24$.

Fig. 5 shows the allowed values for NS mass and radius for redshifts $z=0.24$ and $z=0.35$ compared to various theoretical mass-radius relations. While $z=0.35$ gives radii of $R=9-12 \mathrm{~km}$ for a mass-range of $M=1.4-1.8 \mathrm{M}_{\odot}$, our redshift $z=0.24$ gives larger radii, namely $12-15 \mathrm{~km}$, which corresponds to stiff equations-of-state and excludes mass-radius relations based on exotic matter. 
This result is in line with a recent study of EXO 0748-676 by Özel (2006) using additional observational constraints. With $z=0.35$ she derives minimum values of mass and radius, $M \geq 2.10 \mathrm{M}_{\odot}$ and $R \geq 13.8 \mathrm{~km}$. A reduction of the redshift to $z=0.24$ has a negligible effect on her radius determination but the lower mass limit is reduced to $1.63 \mathrm{M}_{\odot}$.

Acknowledgements TR is supported by BMBF/DESY (grant 05 AC6VTB) and VS by DFG (grant We 1312/35-1) and Russian FBR (grant 05-02-17744).

\section{References}

Cottam, J., Paerels, F., Mendez, M. Gravitational redshifted absorption lines in the X-ray burst spectra of a neutron star. Nature 420, 51-54, 2002.

Ertan, Ü., Cheng, K.S. On the Infrared, Optical, and High-Energy Emission from the Anomalous X-Ray Pulsar 4U 0142+61. ApJ 605, 840-845, 2004.

Ertan, Ü., Erkut, M.H., Eksi, K.Y., Alpar, M.A. A Fallback Disk Around a Neutron Star Spinning Down While Accreting. ApJ in press, astro-ph/0606259, 2006.

Graves, G. J. M., Challis, P. M., Chevalier, R. A., Crotts, A., Filippenko, A. V., Fransson, C., Garnavich, P., Kirshner, R. P., Li, W., Lundqvist, P., McCray, R., Panagia, N., Phillips, M. M., Pun, C. J. S., Schmidt, B. P., Sonneborn, G., Suntzeff, N. B., Wang, L., Wheeler, J. C. Limits from the Hubble Space Telescope on a Point Source in SN 1987A. ApJ 629, 944-959, 2005.

Haensel, P., Potekhin, A.Y., Yakovlev, D.G. Neutron Stars 1. Equation of State and Structure, Springer, Berlin, 2006.

Hulleman, F., van Kerkwijk, M.H., Kulkarni, S.R. The Anomalous X-ray Pulsar $4 \mathrm{U} 0142+61$ : Variability in the infrared and a spectral break in the optical. A\&A 416, 1037-1045, 2004.

Kern, B., Martin, C. Optical pulsations from the anomalous X-ray pulsar 4U0142+61. Nature 417, 527-529, 2002.

Lai, D. Matter in strong magnetic fields. Rev. Mod. Phys. 73, 629-662, 2001.

Lai, D., Salpeter, E.E. Hydrogen Phases on the Surface of a Strongly Magnetized Neutron Star. ApJ 491, 270-285, 1997.

Menou, K., Perna, R., Hernquist, L. Stability and Evolution of Supernova Fallback Disks. ApJ 559, 1032-1046, 2001.

Nagel, T., Dreizler, S., Rauch, T., Werner, K. AcDc - A new code for the NLTE spectral analysis of accretion discs: application to the helium CV AM CVn. A\&A 428, 109-115, 2004.

Özel, F. Soft equations of state for neutron-star matter ruled out by EXO 0748-676. Nature 441, 1115-1117.

Pavlov, G.G., Shibanov, I.A., Zavlin, V.E. Spectra of X-ray bursts at nearEddington luminosities. MNRAS 253, 193-197, 1991. 
Rauch, T., Deetjen, J.L. Handling of Atomic Data, in: Hubeny, I., Mihalas, D., Werner, K. (Eds.), Stellar Atmosphere Modeling. ASP Conf. Series 288, 103-116, 2003.

Suleimanov, V., Werner, K. Importance of Compton scattering for radiation spectra of isolated neutron stars with weak magnetic field. A\&A submitted, 2007.

van Paradijs, J., Taam, R.E., van den Heuvel, E.P.J. On the nature of the 'anomalous' 6-s X-ray pulsars. A\&A 299, L41-44, 1995.

Wang, Z., Chakrabarty, D., Kaplan, D.L. A debris disk around an isolated young neutron star. Nature 440, 772-775, 2006.

Werner, K., Deetjen, J.L. Non-LTE effects in neutron star atmospheres, in: Kramer, M., Wex, N., Wielebinski, R. (Eds.), Pulsar Astronomy - 2000 and beyond. IAU Coll. 177, ASP Conf. Series 202, 623-624, 2000.

Werner, K., Deetjen, J.L., Dreizler, S., et al. Photospheres with Accelerated Lambda Iteration, in: Hubeny, I., Mihalas, D., Werner, K. (Eds.), Stellar Atmosphere Modeling. ASP Conf. Series 288, 31-50, 2003.

Werner, K., Nagel, T., Rauch, T. Non-LTE modeling of supernova-fallback disks. Ap\&SS, in press, astro-ph/0608529 (2006). 